nance. However, unconstrained growth in spending for health care led to a prospective payment system and diagnosisrelated groups (DRGs), thus limiting hospital revenues. As managed care has entered the scene, revenues to hospitals have fallen further; cross-subsidization of health care, education and research is threatened; and more care is being shifted to the ambulatory arena. These factors have caused reductions in hospital admissions, census, length of stay and, ultimately, revenues. The ways in which hospitals have begun to respond and will need to respond are discussed in three different scenarios (Chapters 5-7), which emphasize managing better in difficult times, hospital-physician realignments, and moving from freestanding hospitals to an integrated health plan, the key players being hospital trustees, hospital administrators and staff physicians. The traditional roles and relationships of these three groups must change substantially if they are to share a common institutional vision and participate jointly in the planning and implementation of programs that are necessary if they are to survive and flourish in this new environment.

In the three final chapters the author offers some insights as to how things might take shape over the next 15-25 years: The list includes progress toward universal coverage, hospital mergers and closures, and growth of managed care. It is suggested that a significant moderation in the rate of health-care expenditures is forthcoming and that this will encompass lower cost treatment sites, constraints on freedom of insured patients and a reduced ability of hospitals to cross-subsidize education and research. Ginzberg also predicts the expansion of managed care to Medicaid (and perhaps to Medicare) beneficiaries. The remaining challenges of a growing number of uninsured and underinsured Americans and precisely how Congress and the nation will respond if market forces do not control health care spending remain open. The author leaves us to ponder these issues with him.

The books provide much food for thought on the remarkable transformation taking place in American health care. Continuing change is virtually certain, but much less certain is precisely what the health-care scene will look like 25 years from now - when many baby-boomers will be 75 years old!

Both books provide the opportunity to view a piece of the future through the eyes of thoughtful individuals.

\title{
Human Molecular Genetics
}

\section{by Tom Strachan and Andrew P. Read Wiley-Liss}

ISBN: 0-471-13373-6, 1996, \$44.95

\section{Reviewed by Patricia D. Murphy GeneWISE, P.O. Box 639 \\ Slingerlands, New York 12159-0639, USA}

Often in academic teaching one has the dilemma that some textbooks are too simple and others too pedantic. The reader of this new book entitled "Human Molecular Genetics" by Tom Strachan and Andrew P. Read does not have to face this dilemma. The authors have taken the very difficult and intense subject of molecular genetics and put the flow of information into reasonably sized chunks to be digested by both the casual reader and the student alike. As a resource it is excellent; as a textbook it would be most appropriate for a two semester course where additional topics from the recent literature can be woven in at will.

Besides the usual introductory chapters on the structure and function of genes, basic inheritance patterns and general technology, there are specialty sections on topics of interest such as the relationship of the mitochondrial genome to the nuclear genome, overlapping or embedded genes, structural motifs (that is, leucine zippers and zinc fingers), imprinting and pseudogene/gene families. Unfortunately for the color-blind reader, all the figures in the book are in red and black. This also means that they do not photocopy well for either handouts or overheads for lectures.

The entire chapter on the evolution of the genome ("Footprints of Evolution") is particularly well done and worth reading in its own right, even though as always happens with textbooks, the recent findings by Venter and his group at The Institute for Genome Research (TIGR) have put a new spin on our view of phylogeny. Since publication of the textbook, this group has shocked the genetics world with the finding that the genome of the methane-producing bacteria Methanococcus jannaschii contains more than $50 \%$ of genes that have never been seen before in nature, a finding that does not easily sit with the established thinking on phylogenetics. Other chapters such as those on cancer, animal models (transgenic or knockout mice) and gene therapy provide good introductions to these topics but may need augmentation if used for teaching these concepts. Examples for the cancer chapter alone would include providing a normal karyotype for comparison with the grossly abnormal karyotype shown in Figure 17.6 and covering additional topics such as the relation of telomerase to cancer and the newer models of colon cancer, both of which were unknown at the time of publication of this book and certainly unpredictable at the time of writing. Finally, the clinical side of human molecular genetics is handled reasonably well in two chapters covering "Molecular Pathology" and "Genetic Testing," the latter dealing with diagnostic, presymptomatic and predispositional testing as well as population screening.

The wonderfully entertaining diagram of the Human Genome Program has a "black cloud" representing "genetic heterogeneity" and "irregular inheritance." Those of us who have had the pleasure of living through this era might disagree with the idea that "unusual" genetics is problematic; indeed, the exceptions to the rules are often the ones that teach us the most. The example I would use from my own career is the Fragile $\mathrm{X}$ syndrome, which taught us about triplet repeats and proved to be the model system for a fair number of neurogenetic disorders. Before the molecular resolution of the nature of the mutation(s) in this disease, there was no clear explanation for the phenomenon of "anticipation." All this is now taken very much for granted when in reality it has been a few short years (five to be exact) since these discoveries were made.

Other nice features of the book include an introduction to the Internet and the World Wide Web, which provides the addresses for general sequence and mapping/marker databases. There is also a reasonably comprehensive glossary with cross-reference to figures and tables where appropriate. All in all, this book is an excellent choice to add to one's own library and to share with clinician friends in need of some basic reading. 